\title{
Konform flache Gravitationsfelder
}

\author{
H. Stephani \\ Theoretisch-Physikalisches Institut der Universität Jena*
}

Eingegangen am 1. April 1967

\begin{abstract}
Using properties of an embedding in a flat sixdimensional space, all solutions of the Einstein equations for a perfect fluid or an electromagnetic field are given, which are conformal to a flat space.

Unter Benutzung der Einbettung in einen sechsdimensionalen flachen Raum werden alle konform flachen Lösungen der Einsteinschen Feldgleichungen bestimmt, die das Gravitationsfeld idealer Flüssigkeiten oder elektromagnetischer Felder beschreiben.
\end{abstract}

\section{Einführung und mathematische Grundlagen}

Eine Lösung der Einsteinschen Feldgleichungen

$$
R_{m n}-\frac{1}{2} g_{m n} R=T_{m n}
$$

ist genau dann konform flach, wenn sich der durch

$$
a_{i ; j ; k}-a_{i ; k ; j}=a^{n} R_{n i j k}, \quad R_{i k}=R^{n}{ }_{i n k}
$$

definierte Krümmungstensor in der Form

$$
R_{i j k l}=\frac{1}{2}\left(g_{i k} R_{j l}-g_{j l} R_{i l}-g_{i l} R_{j k}\right)-\frac{R}{6}\left(g_{i k} g_{j l}-g_{i l} g_{j k}\right)
$$

darstellen läßt. Aus der Differentialgeometrie [1] weiß man, daß sich ein solcher konform flacher Raum immer in einen - höchstens - sechsdimensionalen flachen Raum einbetten läßt. Es existieren also zwei symmetrische Tensoren $\Omega_{i k}, V_{i k}$ und ein Vektor $\tau_{j}$, aus denen sich der Krümmungstensor gemäß

$$
R_{i j k l}=e_{1}\left(\Omega_{i k} \Omega_{j l}-\Omega_{i l} \Omega_{j k}\right)+e_{2}\left(V_{i k} V_{j l}-V_{i l} V_{j k}\right)
$$

aufbaut und die den Gleichungen

$$
\begin{gathered}
V_{i k ; j}-V_{i j ; k}=-e_{1}\left(\tau_{j} \Omega_{i k}-\tau_{k} \Omega_{i j}\right) \\
\Omega_{i k ; j}-\Omega_{i j ; k}=e_{2}\left(\tau_{j} V_{i k}-\tau_{k} V_{i j}\right) \\
\tau_{j ; k}-\tau_{k ; j}=\Omega_{j n} V_{k}^{n}-\Omega_{k n} V_{j}^{n}
\end{gathered}
$$

genügen; $e_{1}$ und $e_{2}$ haben den Wert \pm 1 . Lassen sich Gl. (4) und (5) mit $V_{n m} \equiv 0, \tau_{j} \equiv 0$ erfüllen, so ist der Raum schon in einen fünfdimensionalen flachen Raum einbettbar.

* Adresse: 69 Jena, Max-Wien-Platz 1, DDR. 
Zur Gewinnung konform flacher Lösungen gehen wir nun folgenden Weg: Nach Vorgabe des Energie-Impuls-Tensors $T_{m n}$ bestimmen wir aus (1), (3) und (4) die Struktur der Tensoren $\Omega_{i k}$ und $V_{i k}$, indem wir alle Tensoren nach einem geeigneten Tetradensystem entwickeln. Die Gleichungen (5a)-(5c) geben dann Aussagen über Hyperflächennormalität, Scherungsfreiheit usw. der benutzten Basisvektoren, mit deren Hilfe die Gleichungen (3) in einem geeigneten Koordinatensystem gelöst werden können.

Bei dem ersten Teil dieses Programms benutzt man vorteilhaft gewisse „Eichtransformationen": $\Omega_{i k}, V_{i k}$ und $\tau_{j}$ sind nicht eindeutig bestimmt, auch

$$
\begin{aligned}
\bar{\Omega}_{i k} & =a \Omega_{i k}+b V_{i k}, \quad \bar{V}_{i k}=c \Omega_{i k}+d V_{i k} \\
\bar{\tau}_{j} & =(a d-b c) \tau_{j}+e_{1} c a,{ }_{j}+e_{2} d b,{ }_{j}
\end{aligned}
$$

erfüllen (4) und (5), wenn nur

$$
\begin{array}{ll}
e_{1}=\bar{e}_{1} a^{2}+\bar{e}_{2} c^{2} & e_{1} a c+e_{2} b d=0 \\
e_{2}=\bar{e}_{1} b^{2}+\bar{e}_{2} d^{2} & \\
\bar{e}_{1}=e_{1} a^{2}+e_{2} b^{2} & \bar{e}_{1} a b+\bar{e}_{2} c d=0 \\
\bar{e}_{2}=e_{1} c^{2}+e_{2} d^{2} &
\end{array}
$$

gelten. Diese Transformationen entsprechen Drehungen der beiden zum vierdimensionalen Raum senkrechten, im sechsdimensionalen Einbettungsraum liegenden Basisvektoren, vgl. [1], S. 163.

\section{Konform flache Gravitationsfelder idealer Flïssigkeiten}

Der Energieimpulstensor einer idealen Flüssigkeit der Ruhemassendichte $\mu$, dem Druck $p$ und der Vierergeschwindigkeit $u_{i}\left(u_{i} u^{i}=-1\right)$ ist

$$
T_{m n}=(\mu+p) u_{m} u_{n}+p g_{m n} .
$$

Wegen (3) hat der Krümmungstensor die Gestalt

$$
\begin{aligned}
R_{i j k l}=\frac{\mu+p}{2}\left(u_{i} u_{k} g_{j l}+u_{j} u_{l} g_{i k}-u_{i} u_{l} g_{j k}-u_{j} u_{k} g_{i l}\right) & + \\
& +\frac{\mu}{3}\left(g_{i k} g_{j l}-g_{i l} g_{j k}\right) .
\end{aligned}
$$

Da er sich allein aus

mit

$$
\Omega_{i k}=C g_{i k}+D u_{i} u_{k}
$$

$$
\mu=3 e_{1} C^{2}, \quad \mu+p=2 C D e_{1}
$$

aufbauen läßt, liegt die Vermutung nahe, daß sich diese Lösungen schon in einen fünfdimensionalen flachen Raum einbetten lassen. Zum Beweis dieser Vermutung haben wir

$$
\Omega_{i k ; l}=\Omega_{i j ; l}
$$


zu zeigen. KundT und TRÜMPER [2] wiesen nach, daß konform flache Materiefelder

$$
\begin{aligned}
& u_{i ; k}=-\dot{u}_{i} u_{k}+\frac{\Theta}{3}\left(g_{i k}+u_{i} u_{k}\right) \\
& \mu^{\prime}{ }_{k}=-\dot{\mu} u_{k}
\end{aligned}
$$

erfüllen. Hieraus und aus

$$
T_{i ; k}^{k}=p^{p}{ }_{i}+(p+\mu) \dot{u}_{i}+[\dot{p}+\dot{\mu}+(p+\mu) \Theta] u_{i}=0
$$

folgt in der Tat (12). Wir erhalten damit den

Satz 1. Alle konform flachen Gravitationsfelder idealer Flüssigkeiten lassen sich in einen fünfdimensionalen flachen Raum einbetten.

Diese Felder wurden in [3] ausführlich untersucht.

\section{Konform flache Gravitationsfelder mit elektromagnetischem Feld}

Unter Verwendung eines orthogonalen Vierbeins aus drei raumartigen Vektoren $v_{i}, w_{i}$ und $z_{i}$ und einem zeitartigen Vektor $u_{i}$ können wir den Energieimpulstensor eines elektromagnetischen Nichtnullfeldes in der Gestalt

$$
T_{i k}=R_{i k}=\lambda^{2}\left(u_{i} u_{k}-v_{i} v_{k}+w_{i} w_{k}+z_{i} z_{k}\right)
$$

schreiben und die Ansätze

$$
\begin{aligned}
\Omega_{n m}= & A u_{n} u_{m}+2 B u_{(n} v_{m)}+2 C u_{(n} w_{m)}+2 D u_{(n} z_{m)}+E v_{n} v_{m}+ \\
& +2 F v_{(n} w_{m}+2 G v_{(n} z_{m}+H w_{n} w_{m}+2 I w_{(n} z_{m)}+K z_{n} z_{m} \\
V_{n m}= & L u_{n} u_{m}+2 M u_{(n} v_{m)}+2 N u_{(n} w_{m)}+2 P u_{(n} z_{m)}+Q v_{n} v_{m}+ \\
& +2 R v_{(n} w_{m)}+2 S v_{(n} z_{m)}+T w_{n} w_{m}+2 W w_{(n} z_{m)}+Z z_{n} z_{m}
\end{aligned}
$$

machen. Der Krümmungstensor hat die Form

$$
\begin{aligned}
R_{i j k l}= & \lambda^{2}\left(u_{i} u_{k} v_{j} v_{l}+v_{i} v_{k} u_{j} u_{l}-u_{i} u_{l} v_{j} v_{k}-v_{i} v_{l} u_{j} u_{k}\right)+ \\
& +\lambda^{2}\left(w_{i} w_{k} z_{j} z_{l}+w_{j} w_{l} z_{i} z_{k}-w_{i} w_{l} z_{j} z_{k}-z_{i} z_{l} w_{j} w_{k}\right) .
\end{aligned}
$$

Zur Auswertung der aus (4) und (17) folgenden Gleichungen für die Koeffizienten $A, B \ldots Z$ kann man die Identitäten benutzen, die von Collinson [4] explizit angegeben wurden: Für jeden Tensor $T$, der sich gemäß

$$
T_{m n r s}=e\left(t_{m r} t_{n s}-t_{m s} t_{n r}\right)
$$

aus einem symmetrischen Tensor $t_{n r}$ bilden läßt, gilt

$$
D_{m n i k} \equiv \Delta_{r s t l} T_{m n r s} T_{i k t l}=4 \Delta_{r s t l} t_{m r} t_{n s} t_{i t} t_{k l} \text {. }
$$

Dabei ist $\Delta_{r s t l}$ die totalantisymmetrische Matrix mit $\Delta_{\mathbf{1 2 3 4}}=1$; in (19) ist über gleiche Indizes im Sinne einer Matrizenmultiplikation zu summieren. Aus (19) folgen die 20 Identitäten

$$
\begin{aligned}
D_{m n i k} & =0 \quad \text { mnik nicht alle verschieden } \\
D_{\mathbf{2 4 3 1}} & =D_{\mathbf{1 4 2 3}}=D_{\mathbf{3 4 1 2}} .
\end{aligned}
$$


Macht man durch eine Eichtransformation (6) $B$ und durch eine $w-z$ Drehung $P$ zu null und wendet die Identitäten (20) auf (4) an, lassen sich die gesuchten Tensoren relativ leicht bestimmen:

$$
\begin{aligned}
\Omega_{m n} & =E w_{n} w_{m}+G z_{n} z_{m} \\
V_{m n} & =L u_{m} u_{n}+M\left(u_{m} v_{n}+v_{m} u_{n}\right)+Q v_{n} v_{m} \\
e_{2}\left(L Q-M^{2}\right) & =e_{1} E G=\lambda^{2} .
\end{aligned}
$$

Die Codazzi-Gleichungen $(5 \mathrm{a})-(5 \mathrm{c})$ sagen dann aus, daß der Vektor $\tau_{j}$ verschwindet und

$$
\left(v_{n} v_{m}-u_{n} u_{m}\right)_{; j}=\left(w_{n} w_{m}+z_{n} z_{m}\right)_{; j}=0, \quad \lambda^{2}=\text { const. }
$$

gilt; Riccitensor und Krümmungstensor sind kovariant konstant

$$
R_{i j k l ; m}=0 \text {. }
$$

Es handelt sich also um symmetrische und - wegen (22) - zerlegbare Räume [5], deren Metrik die Struktur

$$
d s^{2}=\left(d x^{1}\right)^{2}+g_{22}\left(x^{1}, x^{2}\right)\left(d x^{2}\right)^{2}+g_{33}\left(x_{3}, t\right)\left(d x^{3}\right)^{2}-d t^{2}
$$

hat. Die Gleichungen (17) geben als Lösung

$$
d s^{2}=\left(d x^{1}\right)^{2}+\cos ^{2} \lambda x^{1}\left(d x^{2}\right)^{2}+\cos ^{2} \lambda t\left(d x^{3}\right)^{2}-d t^{2},
$$

d. h. der Raum ist das Produkt zweier zweidimensionaler Räume konstanter Krümmung.

$\mathrm{Zu}$ dieser Metrik läßt sich stets ein elektromagnetisches Feld finden, das bis auf eine Dualitätsrotation bestimmt ist und z. B. die Gestalt

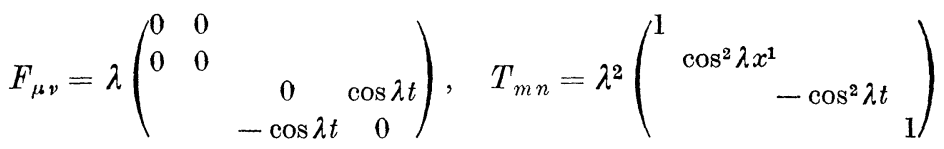

hat. Auch der Feldstärketensor ist kovariant konstant. Wir fassen das Ergebnis zusammen im

Satz 2. Alle konform flachen Gravitationsfelder mit elektromagnetischem Nichtnullfeld sind das Produkt zweier zweidimensionaler Räume konstanter Krümmung; sie haben die Metrik (24). Der elektromagnetische Feldstärketensor ist kovariant konstant und hat die Gestalt (25).

Diese Räume wurden von BerTotTi [6] untersucht.

\section{Konform flache Gravitationsfelder mit elektromagnetischem Nullfeld}

Den Nullvektor $k_{i}$ des Energieimpulstensors

$$
T_{i k}=R_{i k}=\lambda^{2} k_{i} k_{k}
$$

ergänzen wir mit dem Nullvektor $m_{i}$ und zwei raumartigen Vektoren $w_{i}$ und $z_{i}$ zu einem Vierbein. Analog zu der Rechnung für Nichtnullfelder 
kann man die Tensoren $\Omega_{m n}$ und $V_{m n}$ bestimmen, die den Krümmungstensor

$$
R_{i j k l}=\frac{\lambda^{2}}{2}\left(k_{i} k_{k} g_{j l}+k_{j} k_{l} g_{i k}-k_{i} k_{l} g_{j k}-k_{j} k_{k} g_{i l}\right)
$$

aufbauen. Es ergeben sich die folgenden drei Möglichkeiten:

$$
\begin{aligned}
& \Omega_{i n}=A k_{i} k_{n}+H\left(w_{i} w_{n}+z_{i} z_{n}\right) \quad e_{1} A H=\frac{\lambda^{2}}{2} \\
& V_{i n}=T w_{i} w_{n}+Z z_{i} z_{n} \quad e_{1} H^{2}+e_{2} T Z=0 \\
& \Omega_{i n}=A k_{i} k_{n}+K z_{i} z_{n} \quad e_{1} A K=N^{2}=\frac{\lambda^{2}}{2} \\
& V_{i n}=N\left(k_{i} w_{n}+w_{i} k_{n}\right) \quad e_{2}=-1 \\
& \Omega_{i n}=C\left(k_{i} w_{n}+w_{i} k_{n}\right) \quad C^{2}=P^{2}=\frac{\lambda^{2}}{2} \\
& V_{i n}=P\left(k_{i} z_{n}+z_{i} k_{n}\right) \quad e_{\mathbf{1}}=e_{\mathbf{2}}=-\mathbf{1} .
\end{aligned}
$$

Aus den Codazzi-Gleichungen (5) folgt in allen drei Fällen, daß das Nullvektorfeld $k_{i}$ bei geeigneter Normierung kovariant konstant ist

$$
k_{i} ;_{m}=0 \text {. }
$$

Unter Benutzung von (27) kann man, Kundт [7] folgend, die Metrik bestimmen:

$$
\begin{aligned}
d s^{2} & =d x^{2}+d y^{2}+2 d u d v+[\alpha(u, x+y)+\beta(u, x-y)] d u^{2} \\
T_{m n} & =\lambda^{2} k_{n} k_{m}, \quad \lambda^{2}=-H,_{x x}=-H,,_{y y} \\
H & =\alpha(u, x+y)+\beta(u, x-y) .
\end{aligned}
$$

Dieses Strahlungsfeld ist ein Maxwellfeld, wenn Vektoren $p_{i}, q_{i}$ existieren mit

$$
\begin{aligned}
F_{n m} & =k_{n} p_{m}-k_{m} p_{n}, \quad F_{n m}=k_{n} q_{m}-q_{n} k_{m} \\
p_{n} k^{n} & =0, \quad p_{n} p^{n}=q_{n} q^{n}=\lambda^{2} \\
F^{n}{ }_{m ; n} & =F^{* n}{ }_{m ; n}=0
\end{aligned}
$$

vgl. [8]. Mit

$$
p_{i}=\lambda(\cos \varphi, \sin \varphi, 0,0), \quad q_{i}=\lambda(-\sin \varphi, \cos \varphi, 0,0)
$$

gibt dies die Bedingung, daß $\lambda e^{i \varphi}$ eine analytische Funktion von $z=x+i y$ ist und wegen (32) $\lambda^{2}$ eine Lösung der Wellengleichung ist. Mögliche Lösungen sind

$$
\begin{aligned}
\lambda e^{i \varphi} & =a \cos [\omega(1+i) z+b] \quad a, b \text { komplex, } \omega \text { reell } \\
\lambda e^{i \varphi} & =a z+b
\end{aligned}
$$

wobei $a, b$ und $\omega$ beliebige Funktionen von $u$ sind. Wir erhalten damit den 
Satz 3. Alle konform flachen Gravitationsfelder mit elektromagnetischem Nullfeld sind ebenfrontige Wellen mit

$$
\begin{aligned}
& d s^{2}=d x^{2}+d y^{2}+2 d u d v+H d u^{2},-\lambda^{2}=H_{x x}=H_{, y y}
\end{aligned}
$$

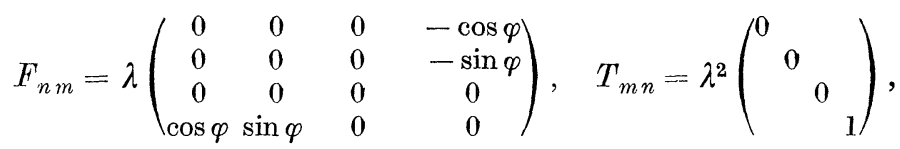

wobei $\lambda e^{i \varphi}$ aus (35) zu entnehmen ist.

Herrn Prof. Dr. Schmutzer und allen Mitgliedern der Arbeitsgruppe Relativitätstheorie danke ich für Anregungen und Diskussionen.

\section{Literatur}

1. Eisenhart, L.: Riemannian Geometry. Princeton: University Press 1926.

2. Kundt, W., u. M. TrÜMPen: Abh. Mainz. Akad. Wiss. 1962, Nr. 12.

3. Stephani, H.: Commun. math. Phys. 4, 137 (1967).

4. Collinson, C. D.: J. Math. Phys. 7, 608 (1966).

5. Petrow, A. S.: Einstein-Räume. Berlin: Akademie-Verlag 1964

6. Bertotti, B.: Phys. Rev. 116, 1331 (1959).

7. Kondt, W.: Z. Phys. 163, 77 (1961).

8. Synge, J. L.: Relativity, the special theory. Amsterdam: North-Holland Publ. Comp. 1956. 